\title{
An Approach of Cloud Based Mobile Learning System (M-learning)
}

\author{
M. Mubasher Hassan ${ }^{1}$ and Tabasum Mirza ${ }^{2}$ \\ Department of Information Technology and Engineering $(I T E)^{1}$ \\ Baba Ghulam Shah Badshah University (BGSBU), Rajouri (J\&K), India \\ Department of Computer Science, School Education ${ }^{2}$ \\ Government of Jammu and Kashmir, J\&K, India \\ mubasher2003@gmail.com ${ }^{1}$, tabasum.mirza@gmail.com ${ }^{2}$
}

\begin{abstract}
In this paper, we are discussing the concept of integrating mobile learning in collaboration with cloud computing and hence giving rise to a reliable approach of mobile learning platform with cost effective features. There are many challenges of developing and managing content for mobile learning at its own like huge initial investment in computing and storage facilities. We can address these issues by hiring the required facilities from any suitable cloud computing vendor. We achieve various types of benefits from cloud computing platform and we can implement a newer version of ICT learning-teaching module called Mobile learning based on cloud computing.
\end{abstract}

Index Terms-Mobile learning, cloud computing, ICT, computing facilities and cost effective.

\section{INTRODUCTION}

Mobile learning has evolved as a popular trend in learner centric learning because of its low cost and portability. Mobile devices are lightweight and portable; they can be carried with ease for communication purpose in the teaching learning system. Mobile learning has some challenges like high initial setup cost, high cost and efforts for content management .Moreover, mobile devices generally have low computational power and storage. Cloud computing can be used to overcome these constraints of mobile learning when integrated with mobile learning to form cloud based mobile learning system that provides benefits like resource pooling, storage of data, collaboration between learners and teachers, low set up cost, backup and recovery of data, flexibility, scalability , accessibility etc. thus making mobile learning effective and powerful. Mobile learning is a powerful learning-teaching tool and can be used as a method to access learning content using mobile devices from anywhere and anytime. It provides continuous access to learning material available with the freedom of space and time. Mobile learning can be asynchronous or synchronous way of learning. Different content types available in different formats make learning interesting and effective. It provides learners with the flexibility of use by providing personalized learning experiences for learners[1].

Cloud computing uses technology like the internet and remote servers to store ,backup, maintenance, management of data, share resources like software and infrastructure, computing power to run applications. It allows businesses and consumers to use applications without installation on client machines by using remote resources, thus saving the cost and increasing the efficiency. It offers ubiquitous, convenient and pervasive access to services that are available remotely on the network, thus increasing productivity of business or consumers[2]. Cloud computing is the delivery of services over the internet where users pay for the services on usage basis or on a subscription basis. The information is stored on physical servers maintained and controlled by cloud computing provider. These systems can be easily scaled up or down anytime depending on the requirements of business. Cloud computing provides secure access to services and data on the go, it also provides auto recovery and backup of data prevents data leakage, unauthorized access and accidental modification of data and disclosure of information[3].

Cloud based systems are mostly used for business or research purpose. It helps businesses to save hardware and software costs by using hardware and software remotely or on the cloud. We can use specialized applications without installation of servers or without the need of installation or buying of hardware or software to run them on any computing device like a laptop, Smartphone, tablet etc.

IAAS: Infrastructure as a service is the cloud based solution or cloud based infrastructure that is outsourced completely e.g. Google, IBM, Rackspace, Amazon EC2 \&Verizon. The different cloud computing services used for effective mobile learning are:

PAAS: Platform as a service provides fully configured deployment and sandbox environment to customers for testing and deployment of cloud applications, mostly used by companies that need to develop, test and deploy cloud solutions resulting in simplified deployment and low cost. Hosting of these applications is done by 3rd party called a PAAS 


\section{International Journal of Research in Advent Technology, Vol.7, No.5, May 2019 E-ISSN: 2321-9637 \\ Available online at www.ijrat.org}

provider, e.g. Rackspace, Google Apps, and MS Azure.

SAAS: Software as a service provides a web based application to customers on demand. The software's can be specialized software having different functionality. This software in SAAS can be free, or payment can be done on usage basis or subscription basis. This provides accessibility from anywhere, rapid scalability and eliminates infrastructure concerns. E.g. Citrix, NetSuite, Webex, Salesforce.

RAAS: Recovery as a service helps companies to achieve backup, archiving, disaster recovery and business continuity in one step and on a single platform that integrates all the functions. It prevents permanent or temporary data loss, including loss of infrastructure, enables fast recovery after a crash. It also reduces costs and improves efficiency, e.g. Geminare, Windstream \& N Scaled.

There are public clouds, which provide service on the internet and are operated by third party companies. They may be free or charged on demand or sold to customers. The private clouds are reserved for usually one private business organization and provided on a private network and the hybrid clouds are a combination of private and public services offering users more flexibility[4].

\section{CONCEPTUAL FRAMEWORK}

The cloud based mobile learning integrates cloud computing and mobile learning and hence giving birth to the better mobile learning facilities. Cloud based mobile learning can make use of cloud computing solutions to make mobile learning flexible and cost effective. The cloud computing can make continuous availability of highly updated resources and quality content possible. The cloud computing can be used for content generation at low cost that can be shared ubiquitously between mobile clients or mobile learners. The cloud computing can help remove obstacles and challenges in the implementation of mobile learning like high initial set up cost, collaboration between different institutions involved, content management, backup and recovery, high storage requirements, high computing power required, high speed internet connectivity, flexibility, accessibility, scalability etc. bringing it as a successful method of teaching and learning[5]. Fig.1. shows the clear pictorial block diagram of cloud based mobile learning.

Content management is the main challenge in mobile learning. Creation and maintenance of high quality, consistent learning resources requires large no. of resources and efforts. It also requires mass storage, high speed internet connectivity and computing resources. Cloud computing can provide mass storage, high computing power and builds high quality learning content on the cloud that can be effectively shared by mobile devices by connecting mobile devices to internet without much effort. The data content can be available in any format like text, video, audio, animations, Pdf etc.

Using cloud computing we can store huge data content, i.e. learning resources with no need to store this data on mobile resources. This data content can be accessed anytime from anywhere using portable devices by providing infrastructure, platform, application, services by cloud[6]. Setting up of mobile learning incurs huge costs and these include hardware costs, software costs and implementation costs. High costs are also incurred in content creation and management. While as cloud based mobile learning provides the opportunity to pool resources and reduces the cost of content management to a large extent. Small institutions can pay subscription charges or usage charges and use services of mobile cloud without the requirement of initial high cost thus making it possible for them to use mobile learning[7]. Cloud based mobile learning provides backup and restoration of data after crashes thus ensuring all time availability of data without failure. It results in improved collaboration between different institutions and learners by providing availability of highly updated data content on the cloud that could be shared between the researchers, developers, teachers and learners[6]. Cloud based mobile learning is highly learner centric and flexible to meet individual requirements of learners without worrying about hardware and other infrastructural issues. These cloud based mobile learning networks are highly scalable and can be scaled up at ease to suit the growing user and infrastructural needs.

\section{BENEFITS}
a. Low Cost
b. Flexibility
c. Accessibility.
d. Scalability.
e. Updated Data content
f. Spontaneous
g. Improved Performance
h. Reliability of data
i. Device independence
j. Pervasive

\section{CHALLENGES}
I. Privacy
II. Security
III. Control
IV. Mobile network connectivity 

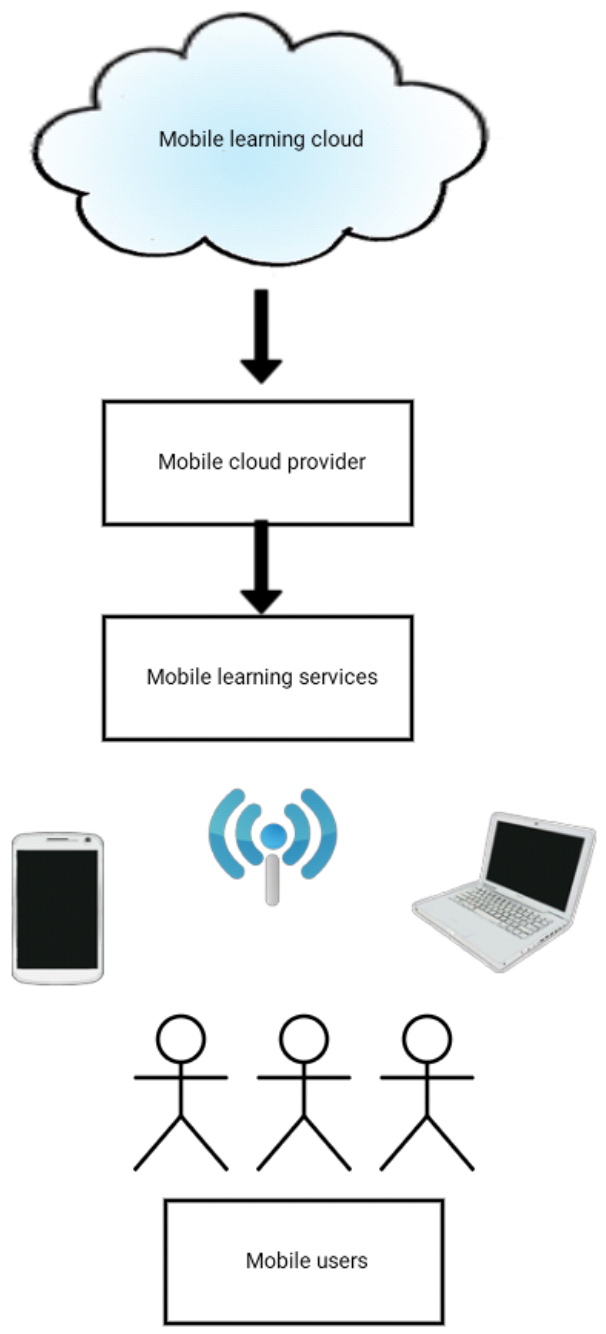

Fig.1. Pictorial view of cloud based M-Learning

\section{CONCLUSION}

It is an established fact that there is a paradigm shift in the teaching-learning processes by introducing different modes of ICT and mobile learning is one among them, which is receiving an increased attention due to its inherent characteristics like easy portability. Although mobile learning can be easily integrated with the already existing e-learning platforms, but to develop an indigenous mobile learning platform requires a secure computing and storage facility. This concept of developing mobile learning facility without investing much on computing resources can be done by cloud computing. The storage space and necessary application software can be hired from reliable vendor of cloud computing and in this way the objective of developing mobile learning facility is achieved in cost effective manner.

\section{REFERENCES}

[1] Y. Mehdipour, "Mobile Learning for Education : Benefits and Challenges," pp. 93-101, 2013.

[2] D. Sullivan and D. Sullivan, "Cloud Computing."

[3] O. Harfoushi, "Influence of Cloud Based Mobile Learning Applications on User Experiences: A Review Study in the Context of Jordan," pp. 202211.

[4] S. Kumar and R. H. Goudar, "Cloud Computing Research Issues, Challenges, Architecture, Platforms and Applications : A Survey," vol. 1, no. $4,2012$.

[5] O. Boyinbode and F. Akintade, "A Cloud Based Mobile Learning Interface," vol. I, pp. 21-24, 2015.

[6] N. M. Rao, "Cloud Computing Through MobileLearning."

[7] S. Dhanalakshmi, S. Suganya, and K. Kokilavani, "International Journal of Computer Sciences Mobile Learning Using Cloud Computing," no. 11, pp. 102-108, 2014. 